\title{
IMPROVING OF ENGINEERING SYSTEM BASED ON FUZZY CONTROL IN MECHANICAL APPLICATION
}

\author{
Mahmoud M. A. Sayed ${ }^{1}$, M.H. Saleh ${ }^{2}$, Tarek A. Madbouly ${ }^{3}$, Mohamed Elhemely ${ }^{4}$ \\ ${ }^{1}$ Canadian International College, Cairo, Egypt \\ ${ }^{2}$ Canadian International College, Cairo, Egypt \\ ${ }^{3}$ Canadian International College, Cairo, Egypt \\ ${ }^{4}$ Canadian International College, Cairo, Egypt
}

\begin{abstract}
The Engineering System (Engsys) depends on many factors such as: inputs, outputs, and automation. This work contributes the linguistic control for industrial applications. Also, it is the development of a linguistic approach for designing Fuzzy Controller "FC" for Engsys. This paper introduced FC in Engsys (robot arm manipulator dynamic) in the mechanical applications, the constant gains for different points not sufficient to satisfy the performance for Engsys manipulator. Therefore, the knowledgebased FC is proposed ether to meet the operating conditions or to remove any fixed mode. The FC uses the error and the change of error as fuzzy linguistic inputs to satisfy the system performance. The proposed programs are developed to simulate the performance of the Engsys. The new controller uses only the available information of the input-output for controlling the position and velocity of the Engsys axes of the motion of the end effectors.
\end{abstract}

Keywords: - Engineering System (Engsys), Fuzzy Control (FC), MATLAB Simulink

\section{INTRODUCTION}

Design of systems may be influenced by types of input/output data. The system can enable the operator to observe the required variables (voltage, current, frequency, power, and the direction of energy) to obtain the output, [1].

The Engsys is a multi-functional manipulator designed to move materials, parts, tools, or specialized devices, through variable programmed motions for the performance. Engsys is a programmable mechanical manipulator, capable of moving along several directions, equipped at its end with a work device called the end effectors or tool and able of performing factory ordinarily done by human beings. The term Engsys is used for a manipulator that has a built-in control system and is capable of stand-alone operation. Also, Engsys is one of the most important machines for industrial automations; flexible multifunctional Engsys can be applied to dangerous environments or routine labor as substitutes for the workers.

Engsys is multivariable nonlinear coupling dynamic systems. Since, the Engsys have complicated nonlinear mathematical models. Control systems based on the model are difficult to design [2-7]. Controlling the position and velocity of the Engsys axes of motion generates the motion of the end effector. An axes of motion in Engsys means a degree of freedom in which the Engsys can move, thus, an n-degree of freedom, manipulator contains n-joints, or in more general terms, n-axes of motion. The axes of motion of the Engsys arm can be either rotary or linear. Engsys is designed in kinematics as a revolute pair, which is simple hinge without sliding. It is driven by an electric motor, which is coupled to Engsys (directly or through a chain or a gear system). Control of an Engsys includes nonlinearities, uncertainties and external perturbations that should be considered. FC is used for monitoring and controls the input scaling factors of the FC according to the actual tracking position error and the actual tracking velocity error [7, 8].

The methodology of FC appears to be very useful when processes are too complex for analysis by conventional techniques. The FC depended controller is independent of either the model structure or the model parameters. This allows simple calculation and low implementation cost which are highly recommended features in Engsys. Due to the operating variables, the constant gains not are sufficient to satisfy the performance for the overall system. Therefore a FC proposed either to meet the operating conditions or to remove any fixed mode [8]. Commonly, in Engsys the human knowledge of the system control is based on the expertise, intuition, knowledge of the system's physical behavior, or a set of subjective goals [9-11].

This paper presents a FC for a nonlinear system to provide robust control characteristics. Also, it is organized as follows; the second section describes the Engsys (robot arm manipulator dynamic). The third section discusses FC scheme. The fourth section is concerned with the design of the proposed adaptive fuzzy control scheme. Finally, the last section concludes analysis of simulation, and conclusions. 


\section{MANIPULATOR DYNAMICS}

Modeling and simulation methodology is able to overcome the problems that may exist in any system by using a certain technique. It can provide the necessary elements about the system (inputs, outputs, and physical interpretation) with monitoring (must describe and identify the variables), [12].

One of the main elements of Engsys is robot arm manipulator dynamic that deals with the analytical study of the geometry of motion of a Engsys arm with respect to a fixed reference of the system without regard to the forces that cause the motion, thus kinematics deals with the analytical description Engsys as a function of time [4, 5].

The problems in Engsys; the first problem is referred to as the forward kinematics problem, while the second is the inverse kinematics (arm solution) problem. Engsys arm dynamics deals with the mathematical formulations of the equations of Engsys arm motion. The dynamic equations of motion of a manipulator are a set of mathematical equations $[5,6]$.

Our study of manipulators focuses on kinematics consideration only. In the first problem, trajectory point is given, (position, velocity, and the acceleration). The second problem is to determine how the Engsys will move as the road selection; the output (motion of manipulator, the position, the velocity, and the acceleration) will be obtained. The problem made up of obtaining dynamic models of the manipulator, and using the models to determine control laws to achieve the desired system response and performance. The dynamic model of multi-link Engsys arm can be described by [3, 4, and 14],

$$
\begin{aligned}
& \mathbf{T}_{\mathbf{i}}=\mathbf{T}_{\mathbf{j}}+\mathbf{T}_{\mathbf{j c}}+\mathbf{T}_{\mathrm{cc}}+\mathbf{T}_{\mathbf{g}} \\
& =\sum^{\mathbf{n}} \mathbf{A}_{\mathrm{ij}} \ddot{\boldsymbol{\theta}}_{\mathbf{j}}+\sum^{\mathbf{n}} \mathbf{B}_{\mathbf{i j}} \ddot{\boldsymbol{\theta}}_{\mathbf{j}}^{2}+\sum_{j=1}^{\mathbf{n} \mathbf{n}} \mathbf{B}_{\mathbf{i j k}} \boldsymbol{\Theta}_{\mathbf{j}} \boldsymbol{\Theta}_{\mathbf{k}}+\mathbf{D}_{\mathbf{i}}
\end{aligned}
$$

Where: $T_{j}, T_{j c}, T_{c c}$ and $T_{g}$ are the torque due to the combined moment of inertia of manipulator and drive part, the torque due to the coupling inertia between the two joint and a gain depend on the angle between, the torque contributed by the centrifugal force due to the velocity in other joint, the torque due to gravity. Matrices $A_{i j}, B_{i j}$, and $\mathrm{B}_{\mathrm{ijk}}$ are the mass, coriolis and centripetal forces which are functions of the joint inertial parameters of the manipulator, and are called the dynamic coefficients of the manipulator; also these are highly complicated nonlinear functions $[3,4]$.

The dynamic model of the Engsys arm excluding the dynamic of the joint motors, backlash, and gear friction can be obtained from lagrange-euler or Newton-euler approach. The dynamic equations of a Engsys:

$$
\mathbf{T}=\mathbf{A}(\boldsymbol{\theta}) \ddot{\boldsymbol{\theta}}+\mathbf{B}(\boldsymbol{\theta}, \ddot{\boldsymbol{\theta}})+\mathbf{C}(\boldsymbol{\theta})
$$

\section{Where; \\ T A generalized vector of joint torques \\ $\boldsymbol{\theta}, \boldsymbol{\Theta} A$ generalized joint coordinate velocity and acceleration vectors \\ $\mathbf{A}(\theta)$ the $n * n$ mass matrix \\ $B(\Theta, \Theta)$ The $n * 1$ vector of centrifugal and coriolis terms \\ $C(\Theta)$ The $n * 1$ nvector of gravity terms}

Each element of $\mathrm{A}(\Theta)$, and $\mathrm{C}(\Theta)$ are complex function which depend on the angle $\Theta$, the position of all the joints of the manipulator, and each element of $\mathrm{B}(\Theta, \Theta)$ is a complex function of both the angle $\Theta$, and the rate of change of the angle $\Theta$. This work computes the dynamic equation of motion for the two-link planner manipulator as shown in Fig.(1)

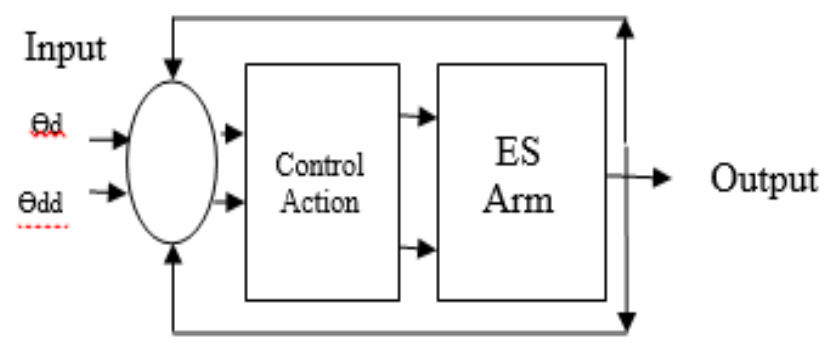

Fig 1: Arm Control of Engsys

The dynamic model of multi-link ES arm can be described by:

$$
T_{1}=A_{11} \ddot{\Theta}_{1}+A_{12} \ddot{\Theta}_{2}+A_{122} \ddot{\Theta}_{2}^{2}+A_{112} \ddot{\Theta}_{1} \ddot{\Theta}_{2}+D_{1} \ddot{\Theta}_{1}+A_{1}
$$

$$
T_{2}=A_{21} \ddot{\Theta}_{1}+A_{22} \ddot{\Theta}_{2}+A_{211} \ddot{\Theta}_{2}^{2}+D_{2} \ddot{\Theta}_{2}+A_{2}
$$

Where:

$A_{1}=m_{1} g s_{1} \sin \theta_{1}+m_{2} g\left(l_{1} \sin \theta_{1}+s_{2}\right.$

$\sin (\theta 1+\theta 2))$

$A_{2}=m_{2} g s_{2} \sin \theta_{2}$

$A_{11}=m_{1} s_{1}{ }^{2}+m_{2}\left(l_{1}{ }_{1}+s_{2}{ }_{2}+2 l_{1} s_{2} \cos \theta_{2}\right)$

$A_{12}=m_{2}\left(s_{2}^{2}+l_{1} s_{2} \cos \theta_{2}\right)$

$A_{122}=-m_{2} l_{1} s_{2} \sin \theta_{2}$

$A_{112}=-2 m_{2} l_{1} s_{2} \sin \theta_{2}$

$\mathbf{A}_{21}=\mathbf{A}_{12}, \quad \mathbf{A}_{22}=\mathbf{m}_{2} \mathbf{s}_{2}{ }^{2}+\mathbf{j}_{\mathrm{m}}$

$A_{211}=m_{2} l_{1} s_{2} \sin \theta_{2}$

$\mathrm{m}_{1}$ is the mass of the link $1, \mathrm{~m}_{2}$ is the mass of the link $2,1_{1}$ is the length of the link $1, \Theta_{1}$ is the angle of the link connection $1, \Theta_{2}$ is the angle of the link connection $2,1_{2}$ is the length of the link 2 ,

The motion equations of a manipulator are coupled and nonlinear second-order ordinary differential equations.

\section{FUZZY CONTROLLER "FC"}

Fig. (2) Shows a FC takes the form of an iteratively adjusting model. The input values are normalized and changed to fuzzy representations, the fuzzy rule produces a 
consequent fuzzy region for each variable, and the consequent regions are defuzzified to obtain the expected value of each variable [8-17].

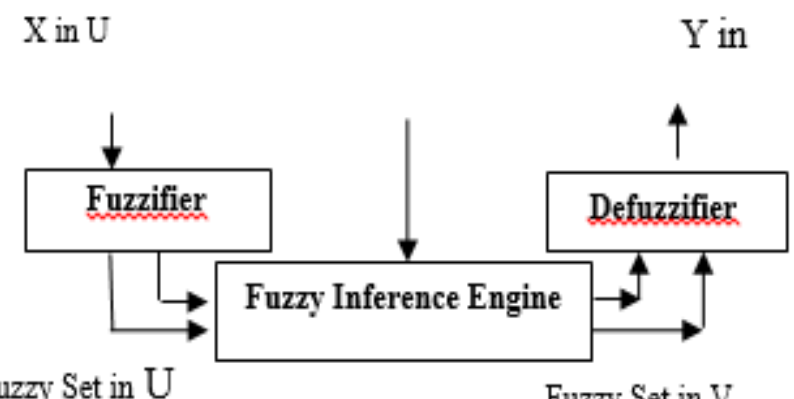

Fuzzy Set in U

Fuzzy Set in V

Fig 2: Fuzzy Controller

FC adjusts its control surface in accord with parameters. Engsys can adapt the changing for normalization of the discourse. FC not only adjusts to time, or process phased conditions, but also changes the supporting system control, $[7,10]$.

\section{PROPOSED FUZZY CONTROLLER}

In this section, the method is conducted the engineers for obtaining the best algorithm that Engsys will work well with the application required.

FC proceeds as follows to evaluate the desired output signal, as shown in Fig.(3). Firstly, the input variables are normalized, and then the membership function of the FC output signal is determined by linguistic codes, finally, the numerical value of FC output signal corresponding to a specific linguistic code is determined.

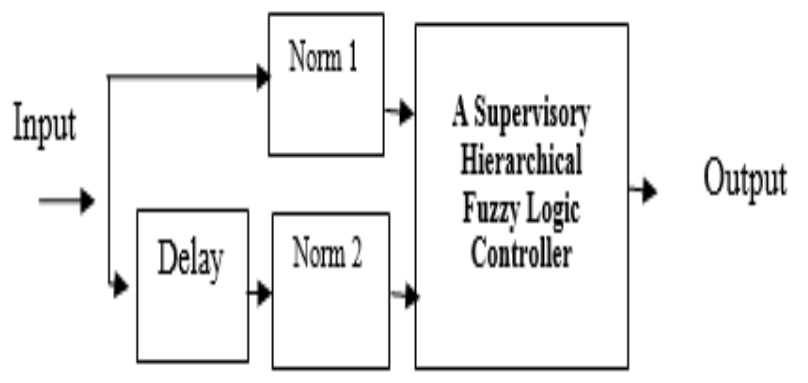

Fig 3: Internal Structure of FC

The programs developed to simulate the dynamic behavior of the Engsys. The new controller uses only the available information of the input-output. FC can obtain the good performance. The simulation have illustrated the effectiveness of the controller in improving drastically controller be used to cope with the possible variation in system parameters. Simulation results using MATLAB SIMULINK show the performance and the same time have removed any error in the resulting scheme.

\section{SIMULATION RESULTS}

Numerical simulations using the dynamic model of a three DOF planar rigid Engsys manipulator with uncertainties show the effectiveness of the controller in set point tracking problems. The results show that the controller has performance compared to a traditional controller. Simulation studies on a pole balancing Engsys and a multilink Engsys manipulator demonstrate the effectiveness and robustness of the proposed approach. In the following, the parameters of a Engsys model are given, each of the physical parameters used in the simulation, where 1 is the length of a link, $s$ is the center of gravity, $m$ is the mass, $D$ is the coefficient of viscous friction, and $\mathrm{j}$ is the moment of inertia, [7, 8, and 18].

The length of link, $1_{1}=1_{2},=0.5 \mathrm{~m}$, the center of gravity, $\mathrm{s}_{1}=$ $\mathrm{s}_{2}=0.25 \mathrm{~m}$,

The mass $: \mathrm{m}_{1}=\mathrm{m}_{2}=0.5 \mathrm{~kg}$, the coefficient of viscous friction $\mathrm{D}_{1}=\mathrm{D}_{2}=0.1 \mathrm{~N} . \mathrm{m} / \mathrm{rad} / \mathrm{s}$,

The moment of inertia $\mathrm{J}=0.1 \mathrm{~kg} \mathrm{~m}^{2}$. Fig.(4) and Fig.(5) show the system response using the proposed Fuzzy Logic Controller technique.
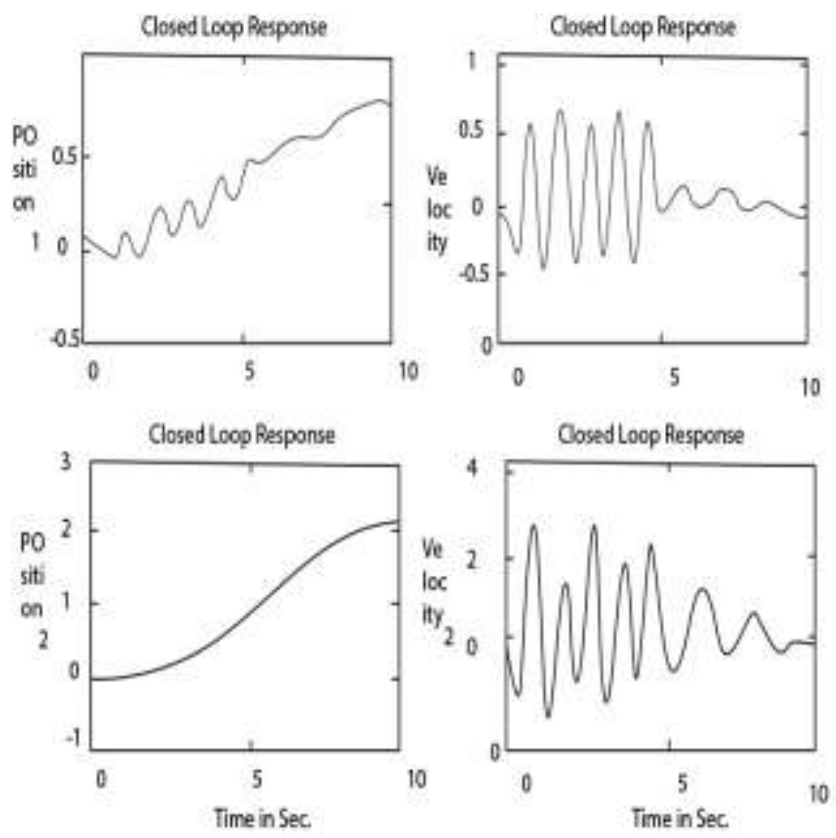

Fig 4: The desired and simulated tracking Positions and Velocities 

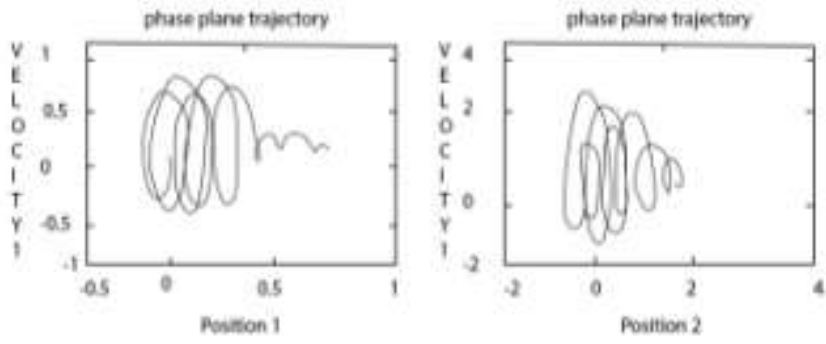

Fig 5: The desired and simulated tracking Positions and Velocities

As it is expected, the FC is faster response with minimum steady state error. This technique should be employed the first peak and the output start decreasing. FC is then independent of either the model structure or the model parameters.

\section{CONCLUSION}

This paper introduced FC of Engsys (robot arm manipulator dynamic) in the mechanical applications, there is an analogous form of in mathematics, where solved a complicated problem in the plant. FC uses the error and the change of error as fuzzy linguistic inputs to regulate the system performance. The proposed programs have been developed to simulate the dynamic behavior of the Engsys. The new controller uses only the available information of the input-output for controlling the position and velocity of the Engsys axes of the motion of the end effectors FC is faster and more accurate. The results validate that the Engsys dynamic response is free speed that improving of Engsys based on FC in mechanical applications.

\section{REFERENCES}

[1] A. M. Fahmy, Hamad M., Mahmoud M. A. Sayed, and Tarek Madbouly, "Smart Bidirectional Energy Meter for A Grid-Interfaced Electric Vehicle Charger", European Journal of Engineering and Technology, Vol. 5 No. 1, 2017.

[2] Aviral Awasthi, SK Gupta and MK Panda "Design of a Fuzzy Logic Controller Based STATCOM for IEEE9 Bus System " European Journal of Advances in Engineering and Technology, 2(4), pp.62-67, 2015.

[3] Ritu Shakya , Kritika Rajanwal, Sanskriti Patel and Smita Dinkar "Design and Simulation of PD, PID and Fuzzy Logic Controller for Industrial Application" International Journal of Information and Computation Technology. Vol. 4, No. 4, pp. 363-368, 2014.

[4] Nizam Uddin Ahamed, Zahari Bin Taha, Mohammad Rabbi, Kenneth Sundaraj "Fuzzy Logic Controller Design for Intelligent Air-Conditioning System", 2nd IEEE International Conference on Control Science and Systems Engineering, July 2016

[5] Kantawong S.," Development of RFID dressing ES using DC servo motor with fuzzy-PID control system " Communications and Information Technologies
(ISCIT), 13th International Symposium. Pp.14-19, 2013

[6] Nanty A., Gelin R., "Fuzzy Controlled PAD Emotional State of a NAO ES " Technologies and Applications of Artificial Intelligence (TAAI) Conference. pp. $90-96,2013$.

[7] Zand, R.M. ; Shouraki, S.B. " Designing a fuzzy logic controller for a quadruped ES using human expertise extraction" Electrical Engineering (ICEE), 21st Iranian Conference, 2013.

[8] Saidon, M.S. ; Desa, H. ; Nagarajan, R. ; Paulraj, M.P. " Vision based tracking control of an autonomous mobile ES in an indoor environment" Control System graduate Research Colloquium (ICSGRC), IEEE, 2011.

[9] Jalali, L., Ghafarian, H., "Maintenance of ES's equilibrium in a noisy environment with fuzzy controller" Intelligent Computing and Intelligent Systems, ICIS, IEEE International Conference, Vol.2. pp. 761-766, 2009.

[10] J.J. Criage, "Introduction to ESics Mechanis and Control" Addison-Wesley Pub Company 1986.

[11] Ahmad N., Al-Husban, "A Learning Linquistic Teaching Control For a Multi-Area Electric Power System" International Journal of Engineering, vol.6, (5), pp.278-284, 2012.

[12] B. Nassar, W. Hussein, and Mahmoud M. A. Sayed, Classification of Multi-failure Mechanisms in Space Operations in Using Novel PLS-DA Approach" IEEE Aerospace Conference, March, 2017.

[13] C.C. Lee, "Fuzzy Logic in Control Systems-Part-I" IEEE Trans. On Systems, Man and Cybernetics, Vol.20, No.2, March/April 1990.

[14] C.C. Lee" Fuzzy Logic in Control Systems-Part-II" IEEE Trans. On Systems, Man and Cybernetics, Vol.20, No.2, March/April 1990.

[15] M.H.Saleh, A.H.Elassal, and I.H.Khalifa, "Fuzzy Logic Controller for Multi-Area Load Frequency Control of Electric Power Systems", Conference on Computer and Applications, IEEE Alex., Sept., 1996.

[16] M.H.Saleh, A.H.Elassal, and I.H.Khalifa, "An Adaptive Fuzzy Controller to Improve System Performance" Conference on Computer and Applications, IEEE Alex., Sept., 1997.

[17] T.Abd El-Rahman, M.H.Saleh "Fuzzy Logic Design Membership Implementation Using Optical Hardware Components" BE-2902R1-Elsevier - 2012.

[18] M.H.Saleh "Fuzzy Logic Design Membership" Ref. 145UPEC2012, 47 International Universities Power Engineering Conference UPEC 2012, Sept., 2012, UK.

[19] Y. Hasegawa, T. Fukada, and K. Shimoojima , “ SelfScaling Reinforcement Learning for Fuzzy Logic Controller-Applications to Motion Control of TwoLink Brachhiation ES" IEEE Tran. On Industrial Electronics, Vol. 46, No.6, Dec., 1999. 